\title{
Is there any gambling disorder among mariners?
}

\author{
Richard Pougnet ${ }^{1,2}$, Laurence Pougnet ${ }^{1,3}$ \\ ${ }^{1}$ Maritime Medicine French Society (SFMM), Brest, France \\ ${ }^{2}$ EA 4686 Ethic Professionalism and Health, European University of Brittany, Brest, France \\ ${ }^{3}$ Military Hospital Clermont-Tonnerre, CC41 BCRM, Brest, France
}

Gambling is listed in the Substance Use and Related Disorders section of the Diagnostic and Statistical Manual of Mental Disorders, Revision V (DSM-V) [1]. In typical clinical case, patient is preoccupied with gambling; he/she needs to gamble with increasing amounts of money; he/she repeats unsuccessful efforts to control, cut back or stop gambling; he/she is restless or irritable when trying to cut down or stop gambling; he/she gambles as a way of escaping from problems or gambles to be relieved; after losing he/she goes on gambling to win money back; he/she lies to family members, therapist or others; he/she has committed illegal acts; he/she has jeopardised relationships or career; he/ /she relies on others to provide bailout [2].

The prevalence of this addiction disorder varies according the study and the country. For example, prevalences of gambling were $0.2 \%$ in Netherlands in 2011 and 41.8\% in a South Korean study in 2014 [3, 4]. The prevalence in lifetime is about $2 \%$ in United States [1]. But it has increased since a few years because of the development of online games $[5,6]$. In some countries this prevalence is constant because of control policies [3]. Given the importance of psychoactive substance consumption among mariners [7], we asked the question whether pathological gambling was a health problem in this population or not, since subjects already having an addiction are more at risk for pathological gambling than the general population [8].

We have done a literature review. Main medical databases, Medline, Embase and Scopus, were searched for relevant information. There wasn't any language restriction. Used keywords were: “Gambling”[Mesh] AND (“'Ships/manpower"[Mesh]) OR ("seafarer* " OR "seamen" OR "seaman" OR "sailor* $\mathrm{OR}$ "mariner*»)). There was no article about gambling among mariners. Yet, it is an historical health problem among this population [9].

However, several arguments let us think that seafarers may be especially susceptible to this addiction.

Firstly, the mariners have a way of life with alternating periods of work and time ashore. During stops or between voyages, they can have access to games in countries with different control policies. But also, the online game may be a problem depending on ship: for example, if they can connect to game websites. We know that the prevalence of pathological gambling with online games was 1.5\% in 2014 in South Korea [4].

It should be noted that the attitude towards gaming differs between cultures. However, gambling disorder develops when this playful relationship is exceeded. We know for example the importance of social games in Asia [5, 10]. That is why gambling disorders and their prevention are different from one country to another and from one culture to another $[11,12]$. The problem is that the mariners live in an international context: various origins of marines, ports of call around the world. Are social conventions and cultural norms effective for a mariner abroad, inside another culture and subjected to multiple stimulations?

In addition, the pathophysiology of gambling disorder is not always clear. Several authors, however, believe that this is a defence strategy initially [10]. That's why anxiety and mood disorders are often described among gambling addicts [13]. These disorders are often described among mariners too. Moreover, between $70 \%$ and $80 \%$ addicts are men [14]. Mariners are mostly men too.

Finally, and more specifically, some personality models describing gambling addicts tend to make us think that mariners are likely to develop this addiction. Access to gambling is an integral component of the Blaszczynski and Nower [15] pathways model. This model incorporates a biopsychosocial approach to conceptualise gambling disorder. The authors thought that all gamblers experience a degree of behavioural conditioning. Besides, there is a development of faulty cognitions, such as overestimating the amount of control over random events and trying to recoup lost money. According to this model, individual vulnerability is an important additional pathway into gambling disorder,

Dr Richard Pougnet, Environmental, Maritime and Professional Pathologies Centre, University Hospital of Morvan, 10, rue des onze Martyrs 29200 Brest, France,

tel: 33601964928, e-mail: richard.pougnet@live.fr 
such as mood and anxiety disorders, trauma or poor coping skills. Another important factor is the impulsivity. This fact reminds us some mariners we saw in our medical centre. It is why we believe that mariners are likely to develop a gambling disorder.

We think it is very important to screen this pathology among mariners. Indeed, gambling disorder is associated with high levels of shame and stigma and most people hide their addiction in order to maintain their job. How can we screen this disorder? A non-judgemental and empathic approach should be adopted when identifying gambling problems [2]. A lot of clinical features can indicate the problem, such as: addiction, mood or anxiety disorders, disrupted sleep, changed eating patterns, dissatisfaction with quality of life $[2,8,14]$. Moreover, standardised questionnaire can be used, such as the South Oaks Gambling Screen (SOGS), the 3-item Brief Bio-Social Gambling Screen or the NODS-CLiP [16-18].

Because of all these arguments, we think that mariners need routine screening for this addiction. So we invite all marine doctors to do the screening of this public health problem.

\section{REFERENCES}

1. Petry NM, Blanco C. National gambling experiences in the United States: will history repeat itself? Addiction 2013; 108: 1032-1037.

2. Rodda S, Lubman DI, Latage K. Problem gambling: aetiology, identification and management. Aust Fam Physician 2012; 41: 725-729.

3. Goudriaan AE. Gambling and problem gambling in The Netherlands. Addiction 2014; 109: 1066-1071.

4. Williams RJ, Lee CK, Back KJ. The prevalence and nature of gambling and problem gambling in South Korea. Soc Psychiatry Psychiatr Epidemiol 2013; 48: 821-834.

5. van den Bos R, Davies W, Dellu-Hagedorn F et al. Cross-species approaches to pathological gambling: a review targeting sex differences, adolescent vulnerability and ecological validity of research tools. Neurosci Biobehav Rev 2013; 37 (10 Part 2): 2454-2471.

6. Jiménez-Murcia S, Fernández-Aranda F, Granero R, Menchón JM. Gambling in Spain: update on experience, research and policy. Addiction 2014; 109: 1595-1601.

7. Pougnet $R$, Pougnet $L$, Loddé $B$ et al. Consumption of addictive substances in mariners. Int Marit Health 2014; 65: 199-204.

8. Lorains FK, Cowlishaw S, Thomas SA. Prevalence of comorbid disorders in problem and pathological gambling: systematic review and meta-analysis of population surveys. Addiction 2011; 106 : 490-498.

9. Taylor P. Spanish Seamen in the New World during the Colonial Period. The Hispanic American Historical Rev 1922; 5: 631-661.

10. Liu L, Luo T, Hao W. Gambling problems in young people: experience from the Asian region. Curr Opin Psychiatry 2013; 26: 310-317.

11. Binde P. Gambling in Sweden: the cultural and socio-political context. Addiction 2014; 109: 193-198.

12. Benegal V. Gambling experiences, problems and policy in India: a historical analysis. Addiction 2013; 108: 2062-2067.

13. Park S, Cho MJ, Jeon $\mathrm{HJ}$ et al. Prevalence, clinical correlations, comorbidities, and suicidal tendencies in pathological Korean gamblers: results from the Korean Epidemiologic Catchment Area Study. Soc Psychiatry Psychiatr Epidemiol 2010; 45: 621-629.

14. Erbas B, Buchner UG. Pathological gambling: prevalence, diagnosis, comorbidity, and intervention in Germany. Dtsch Arztebl Int, 2012; 109: 173-179.

15. Blaszczynski A, Nower L. A pathways model of problem and pathological gambling. Addiction 2002; 97: 487-499.

16. Lesieur HR, Blume SB. The South Oaks Gambling Screen (SOGS): a new instrument for the identification of pathological gamblers. Am J Psychiatry 1987; 144: 1184-1188.

17. Gebauer L, LaBrie R, Shaffer H. Optimizing DSMIV-TR classification accuracy: a brief biosocial screen for detecting current gambling disorders among gamblers in the general household population. Can J Psychiatry 2010; 55: 82.

18. Toce-Gerstein M, Gerstein D, Volberg R. The NODS-CLiP: a rapid screen for adult pathological and problem gambling. J Gambl Stud 2009; 25: 541-555. 